\title{
WHITEHEAD PRODUCTS IN THE COMPLEX STIEFEL MANIFOLDS
}

\author{
by YASUKUNI FURUKAWA
}

(Received 5th January 1982)

\section{Introduction}

The complex Stiefel manifold $W_{n, k}$, where $n \geqq k \geqq 1$, is a space whose points are $k$-frames in $C^{n}$. By using the formula of McCarty [4], we will make the calculations of the Whitehead products in the groups $\pi_{*}\left(W_{n, k}\right)$. The case of real and quaternionic will be treated by Nomura and Furukawa [7]. The product $\left[[\eta], j_{1} l\right]$ appears as generator of the isotropy group of the identity map of Stiefel manifolds. In this note we use freely the results of the 2-components of the homotopy groups of real and complex Stiefel manifolds such as Paechter [8], Hoo-Mahowald [1], Nomura [5], Sigrist [9] and Nomura-Furukawa [6].

\section{Preliminaries}

We identify $W_{n, k}$ with $S U(n) / S U(n-k)$ in the usual way, and consider the boundary homomorphism $\Delta: \pi_{r+1}\left(W_{n, k}\right) \rightarrow \pi_{r}(S U(n-k))$ in the homotopy sequence of a fibration $S U(n) \rightarrow W_{n, k}$. A homomorphism $P_{k}^{c}$ is defined by requiring that the diagram

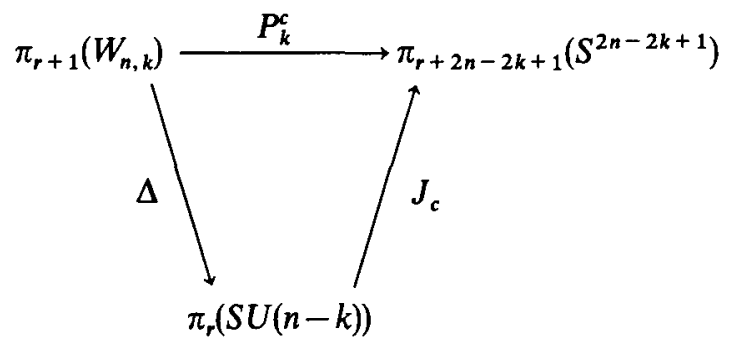

commutes where $J_{c}$ is given by the McCarty product [4], $J_{c}(\gamma)=\left\langle\gamma, l_{2 n-2 k+1}\right\rangle$ for $\gamma \in \pi_{r}(S U(n-k))$. Since the inclusion $j_{k-1}: S^{2 n-2 k+1} \rightarrow W_{n, k}$ is $S U(n-k)$-equivariant, we have

Lemma 1. Let $\alpha \in \pi_{r+1}\left(W_{n, k}\right)$. Then we have $\left[\alpha, j_{k-1} l_{2 n-2 k+1}\right]= \pm j_{k-1} P_{k}^{c}(\alpha)=$ $\pm j_{k-1} E J i \Delta(\alpha)$, where $i: S U(n-k) \rightarrow R_{2 n-2 k}$ is the map into the group of rotations. 
Proof. By McCarty [4], we have

$$
\begin{aligned}
{\left[\alpha, j_{k-1} l_{2 n-2 k+1}\right] } & = \pm\left\langle\Delta \alpha, j_{k-1} l_{2 n-2 k+1}\right\rangle \\
& = \pm j_{k-1}\left\langle\Delta \alpha, l_{2 n-2 k+1}\right\rangle \\
& = \pm j_{k-1} P_{k}^{c}(\alpha) \\
& = \pm j_{k-1} J_{c} \Delta(\alpha) \\
& = \pm j_{k-1} \operatorname{EJi\Delta }(\alpha) .
\end{aligned}
$$

The following diagram commutes;

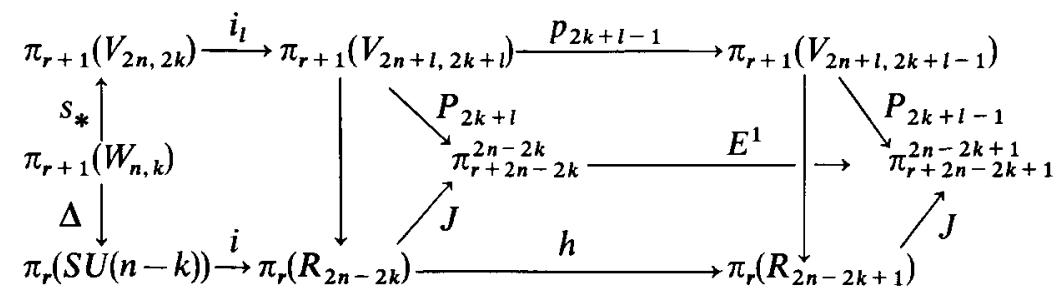

where $s, i_{l}$ and $h$ are the inclusions and $p_{2 k+l-1}$ is the projection. Denote by $s_{k}$ a cross section of a fibration $W_{n, k} \stackrel{q_{1}}{\longrightarrow} S^{2 n-1}$ if it exists and $[\gamma]$ an element of the group $\pi_{*}\left(W_{n, k}\right)$ such that $q_{1 *}[\gamma]=\gamma$ for an element $\gamma \in \pi_{*}\left(S^{2 n-1}\right)$. For the real Stiefel fibration $V_{2 n, k} \stackrel{p_{1}}{\longrightarrow} S^{2 n-1}$, we use the same notations. For $m<2 k$, there is the commutative diagram;

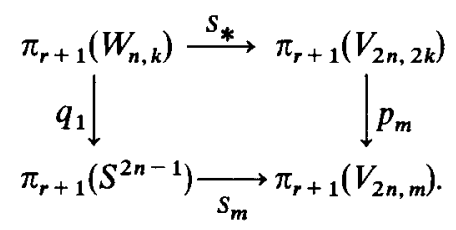

Let $\Delta_{k-1}: \pi_{r+1}\left(W_{n, k-1}\right) \rightarrow \pi_{r}\left(S^{2 n-2 k+1}\right)$ denote the boundary homomorphism in the homotopy sequence of a fibration $S^{2 n-2 k+1} \stackrel{j_{k-1}}{\longrightarrow} W_{n, k} \rightarrow W_{n, k-1}$. For $\Delta_{k-1}$, we have the following :

Lemma 2. (i) Let $\alpha \in \pi_{r}\left(S^{2 n-2}\right)$. Then $\Delta_{1}(E \alpha)=0$ for $n$ even, $\Delta_{1}(E \alpha)=\eta_{2 n-3} \alpha$ for $n$ odd. (ii) Let $n$ be even, then $\Delta_{2} j_{1^{*}} E \alpha=\eta_{2 n-5^{\alpha}} \alpha$ for $\alpha \in \pi_{r}^{2 n-4}, \Delta_{2} s_{2^{*}} E \gamma= \pm b_{n}\left(v_{2 n-5}+\alpha_{1}\right) \gamma$ for $\gamma \in \pi_{r}^{2 n-2}$.

(iii) Let $n$ be odd, then $\Delta_{2} j_{1^{*}} E \alpha=0$ for $\alpha \in \pi_{r}^{2 n-4}, \Delta_{2}\left\{j_{1^{*}}, \eta, \gamma\right\} \equiv-a_{n}\left(v+\alpha_{1}\right) \gamma \bmod a_{n}(2 v$ $\left.+\alpha_{1}\right) \gamma$, where $a_{n}=(12,(n-3) / 2), b_{n}=(12, n / 2)$. 


\section{Whitehead products in $\pi_{r}\left(W_{n, 2}\right)$ for $n$ odd}

For the homotopy groups of $W_{n, k}$, see [6] and [9]. Let $n$ be odd. The group $\pi_{2 n-1}\left(W_{n, 2}\right)=Z$ is generated by $[2 l]$ with $q_{1 *}[2 l]=2 l$.

Theorem 3. We have $\left[[2 l], j_{1} l_{2 n-3}\right]=0$.

Proof. Let $k=2, l=1$ and $r=2 n-2$ in (1.1). Since the group $\pi_{2 n-1}\left(V_{2 n+1,5}\right)=Z_{4}$ is generated by $i_{4} v$, we have $p_{4} \pi_{2 n-1}\left(V_{2 n+1,5}\right)=0$. This implies

$$
\begin{aligned}
{\left[[2 l], j_{1} l_{2 n-3}\right] } & = \pm j_{1} \operatorname{EJi\Delta }[2 l] \\
& = \pm j_{1} P_{4} p_{4} i_{1} s_{*}[2 l] \\
& =0 .
\end{aligned}
$$

The 2-component of the group $\pi_{2 n+2}\left(W_{n, 2}\right)$ is $Z_{8}$ generated by [ $\left.v\right]$.

Theorem 4. We have $\left[[v], j_{1} l_{2 n-3}\right]= \pm j_{1} E P_{4}[v] \neq 0$ for $n \equiv 1 \bmod 4$, and 0 for $n \equiv 3 \bmod 4$.

Proof. Let $k=2, l=4$ and $r=2 n+1$ in (1.1). The group $\pi_{2 n+2}\left(V_{2 n, 4}\right)=Z_{2}+Z_{8}$ is generated by $i_{3} v^{2}$ and $[v]$, so we may set $s_{*}[v] \equiv[v] \bmod i_{3} v^{2}$ by $(1.2)_{2}$. By Lemma 1 , we have

$$
\begin{aligned}
{\left[[v], j_{1} l_{2 n-3}\right] } & = \pm j_{1} P_{7} p_{7} i_{4} s_{*}[v] \\
& = \pm j_{1} E P_{4}[v] \\
& = \pm j_{1} P_{3}[v] .
\end{aligned}
$$

Consider the commutative diagram

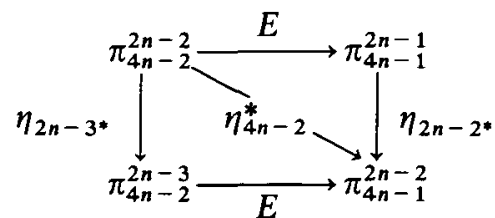

If $n \equiv 3 \bmod 4$, we have

$$
E \eta_{2 n-3^{*}} P_{4} s_{4} l_{2 n+1}=\eta_{4 n-2}^{*} P_{4} s_{4} l_{2 n+1}=P_{4} s_{4} \eta_{2 n+1}=E P_{5} s_{5} \eta
$$

for $P_{4} s_{4} l_{2 n+1} \in \pi_{4 n-2}^{2 n-2}$. This shows that $\eta_{2 n-3^{*}} P_{4} s_{4} l_{2 n+1}=P_{5} s_{5} \eta_{2 n+1}$, since $E: \pi_{4 n-2}^{2 n-3} \rightarrow \pi_{4 n-1}^{2 n-2}$ is the monomorphism. Since $i_{4}[v]=i_{2} s_{5} \eta$ we have

$$
P_{3}\left[v_{2 n-1}\right]=P_{5} s_{5} \eta_{2 n+1} \in \operatorname{Image} \Delta_{1} \text {. }
$$


Therefore $\left[[v], j_{1} \imath_{2 n-3}\right]=0$.

If $n \equiv 1 \bmod 4$, we have $0 \neq\left[v, \imath_{2 n-1}\right]=E^{3} P_{4}[v]$ and $H_{1} P_{4}[v]=v^{2}$ by [3]. This shows $E P_{4}[v] \notin$ Image $E^{2}$. The unstable parts $Z_{8}+Z_{24}$ of the group $\pi_{4 n-2}^{2 n-2}$ which is generated by $P_{4} s_{4} l$ and $\left[v, l_{2 n-2}\right]$ vanish by $\eta_{4 n-2}^{*}$, since $H_{5}\left[l_{2 n+3}, l_{2 n+3}\right]=i_{1} s_{4} \eta$ implies $P_{4} s_{4} \eta=0$. Therefore the unstable element $P_{3}[v]$ does not lie in $\eta_{2 n-3^{*}} \pi_{4 n-2}^{2 n-2}=$ Image $\Delta_{1}$. This shows that

$$
\left[[v], j_{1} l_{2 n-3}\right]= \pm j_{1} P_{3}[v] \neq 0
$$

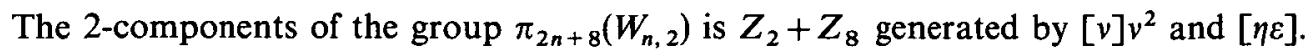

Theorem 5. We have $\left[[\eta \varepsilon], j_{1} l_{2 n-3}\right]= \pm 4 j_{1} P_{5}[\sigma]$ for $n \equiv 1 \bmod 4$ and $n \equiv 27 \bmod 64$, $\left[[\eta \varepsilon], j_{1} l_{2 n-3}\right]=0$ otherwise.

Proof. Let $k=2, l=5$ and $r=2 n+7$ in (1.1). The group $\pi_{2 n+8}\left(V_{2 n, 4}\right)=Z_{2}+Z_{4}$ is generated by $[v] v^{2}$ and $[\eta \varepsilon]$, so we may set $s_{*}[\eta \varepsilon]=[\eta \varepsilon]$ in $(1.2)_{2}$. By the result of the groups $\pi_{2 n+8}\left(V_{2 n+5, k}\right)$ for $k=8$ and 9 , we have the following:

$$
\begin{aligned}
& {\left[[\eta \varepsilon], j_{1} \imath_{2 n-3}\right]= \pm 4 j_{1} P_{5} s_{5} \sigma \text { for } n \equiv 3 \bmod 4,} \\
& {\left[[\eta \varepsilon], j_{1} \imath_{2 n-3}\right]= \pm 4 j_{1} P_{5}[\sigma] \text { for } n \equiv 1 \bmod 4}
\end{aligned}
$$

If $n \equiv 3 \bmod 4$, except $n \equiv 27 \bmod 64$, the relations

$$
H_{12} P_{1} l_{2 n+9}=\lambda i_{7} s_{5} \sigma(\lambda=0,1,2,4)
$$

lead to $\left[[\eta \varepsilon], j_{1} l_{2 n-3}\right]=0$.

\section{Whitehead products in $\pi_{r}\left(W_{n, 3}\right)$ for $n$ even}

Let $n$ be even. The group $\pi_{2 n-1}\left(W_{n, 3}\right)=Z$ is generated by $\left[\left(12 / b_{n}\right) 1\right]$.

Theorem 6. The nontrivial Whitehead product $\left[\left[\left(12 / b_{n}\right) r\right], j_{2} l_{2 n-5}\right]$ is equal to $\pm j_{2} P_{3}\left[\eta_{2 n-3}^{2}\right]$ for $n \equiv 2 \bmod 4, \pm j_{2} P_{4}\left[\eta_{2 n-2}\right]$ for $n \equiv 4 \bmod 8, \pm j_{2} P_{5} s_{5} l_{2 n-1}$ for $n \equiv 0$ mod 8 , respectively.

Proof. Let $k=3, l=1$ and $r=2 n-2$ in (1.1). In (1.2) $)_{3}$ the group $\pi_{2 n-1}\left(V_{2 n, 6}\right)=Z_{8}+Z$ is generated by $i_{1}[\eta]$ and $[2 l]$ (or $s_{6} l_{2 n-1}$ for $n \equiv 0 \bmod 4$ ), so we may set

$$
\begin{gathered}
s_{*}\left[\left(12 / b_{n}\right) l\right] \equiv\left(6 / b_{n}\right)[2 l] \bmod 4 i_{1}[\eta] \text { for } n \equiv 2 \bmod 4, \\
s_{*}\left[\left(12 / b_{n}\right) l\right] \equiv\left(12 / b_{n}\right) s_{6} l_{2 n-1} \bmod 4 i_{1}[\eta] \text { for } n \equiv 0 \bmod 4 .
\end{gathered}
$$

If $n \equiv 2 \bmod 4$, the relations $E^{2} P_{3}\left[\eta_{2 n-3}^{2}\right]=\left[\eta^{2}, l_{2 n-3}\right]=E^{6} P_{7}\left[\eta^{2}\right], H_{1} P_{7}\left[\eta^{2}\right]=\varepsilon_{2 n-10}$ and $2 i_{2}[\eta]=-2 i_{1}[2 l]=i_{3}\left[\eta^{2}\right]$ in $\pi_{2 n-1}\left(V_{2 n+1,6}\right)$ imply that $P_{3}\left[\eta^{2}\right]$ is the unstable 
element $2 P_{4}[\eta]=-2 P_{5}[2 l]$ of the group $\pi_{4 n-7}^{2 n-5}=G_{2 n-2}+Z_{8}$, where $Z_{8}$ is generated by $P_{4}[\eta]$ (cf. [10]). Thus

$$
\begin{aligned}
{\left[\left[\left(12 / b_{n}\right) l\right], j_{2} l_{2 n-5}\right] } & = \pm j_{2} P_{6} p_{6} i_{12}[2 l] \\
& = \pm j_{2} P_{3}\left[\eta^{2}\right] \bmod j_{2} 2 P_{3}\left[\eta^{2}\right]=0
\end{aligned}
$$

since $2 P_{3}\left[\eta^{2}\right] \in \operatorname{Im} \Delta_{2}$.

Now the unstable parts $Z_{2}+Z_{2}$ of the group $\pi_{4 n-7}^{2 n-4}$ (cf. [10]) are generated by $P_{2} s_{2} \eta_{2 n-3}$ and $\left[\eta^{2}, l_{2 n-4}\right]$. So we have

$$
\eta_{2 n-5 *} \pi_{4 n-7}^{2 n-4} \not \supset P_{3}\left[\eta^{2}\right]
$$

because of $\eta_{2 n-5^{*}} P_{2} s_{2} \eta_{2 n-3}=P_{2}[\eta] \eta^{2}=2 P_{3}\left[\eta^{2}\right]$. Hence $P_{3}\left[\eta^{2}\right] \notin$ Image $\Delta_{2}$. Thus $j_{2} P_{3}\left[\eta^{2}\right] \neq 0$.

For the case $n \equiv 4 \bmod 8$, the relations $E^{3} P_{4}\left[\eta_{2 n-2}\right]=\left[\eta, l_{2 n-2}\right]=E^{7} P_{8}[\eta]$ and $H_{1} P_{8}[\eta]=\eta \sigma$ show that the element $P_{4}[\eta]$ is the unstable element $2 P_{5} s_{5} l_{2 n-1}$ of the group $\pi_{4 n-7}^{2 n-5}=G_{2 n-2}+Z_{16}$, where $Z_{16}$ is generated by $P_{5} s_{5} l_{2 n-1}$ (cf. [10]). So

$$
\eta_{2 n-5} \pi_{4 n-7}^{2 n-4} \not \supset P_{4}[\eta]
$$

since $\eta_{2 n-5 *} P_{2} s_{2} \eta_{2 n-3}=P_{2}[\eta] \eta^{2}=8 P_{5} s_{5} l_{2 n-1}$.

An argument similar to the above one shows that $\pm b_{n} v_{2 n-5 *} \pi_{4 n-7}^{2 n-2} \not \nexists P_{4}[\eta]$. Therefore we may conclude that

$$
\begin{aligned}
{\left[\left[\left(12 / b_{n}\right) l\right], j_{2} l_{2 n-5}\right] } & = \pm j_{2} P_{6} p_{6} i_{1} 2 s_{6} l \\
& = \pm j_{2} P_{6} p_{6} i_{2}[\eta] \\
& = \pm j_{2} P_{4}[\eta] \\
& \neq 0 \bmod 4 j_{2} P_{4}[\eta]=0,
\end{aligned}
$$

since $\eta_{2 n-5} \pi_{4 n-7}^{2 n-4} \ni 4 P_{4}[\eta]$.

If $n \equiv 0 \bmod 8$, the relations

$$
\eta_{2 n-5^{*}} P_{2} s_{2} \eta_{2 n-3}=P_{2}[\eta] \eta^{2}=4 P_{4}[\eta]=8 P_{5} s_{5} l_{2 n-1}
$$

imply $P_{5} s_{5} l_{2 n-1} \notin \eta_{2 n-5} \pi_{4 n-7}^{2 n-4}$. Assume that $P_{5} s_{5} l \in b_{n} v_{2 n-5} \pi_{4 n-7}^{2 n-2}$. Then we have a contradiction

$$
0 \neq P_{5} s_{5} \eta=\eta_{4 n-7}^{*} P_{5} s_{5} l \in \eta_{4 n-7}^{*} b_{n} v_{2 n-5} \pi_{4 n-7}^{2 n-2}=0
$$


Hence

$$
\begin{aligned}
{\left[\left[\left(12 / b_{n}\right) l\right], j_{2} l_{2 n-5}\right] } & = \pm j_{2} P_{6} p_{6} i_{1} s_{6} l \\
& = \pm j_{2} P_{5} s_{5} l \\
& \neq 0 \bmod 4 j_{2} P_{4}[\eta]=0 .
\end{aligned}
$$

We know that the 2 components of the group $\pi_{2 n}\left(W_{n, 3}\right)$ is $Z_{2}+Z_{8}$, where $Z_{2}$ is generated by $[\eta]$.

\section{Theorem 7. $\left[[\eta], j_{2} l_{2 n-5}\right]=0$.}

Proof. Let $k=3, l=1$ and $r=2 n-1$ in (1.1). In the case $n \equiv 0 \bmod 4$, the group $\pi_{2 n}\left(V_{2 n, 6}\right)=Z_{2}+Z_{2}+Z_{2}+Z_{2}$ is generated by $i_{5} v^{2}, i_{2}[v], i_{1}[\eta] \eta$ and $s_{6} \eta_{2 n-1}$, so we may set

$$
s_{*}[\eta] \equiv s_{6} \eta_{2 n-1} \bmod i_{5} v^{2}
$$

In the following diagram, $E$ is monomorphism

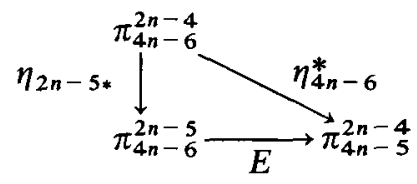

so the relations

$$
E \eta_{2 n-5^{*}} P_{4} s_{4} l_{2 n-1}=\eta_{4 n-6}^{*} P_{4} s_{4} l_{2 n-1}=P_{4} s_{4} \eta_{2 n-1}=E P_{5} s_{5} \eta_{2 n-1}
$$

imply $P_{5} s_{5} \eta_{2 n-1} \in \eta_{2 n-5} * \pi_{4 n-6}^{2 n-4}$. Thus

$$
\begin{aligned}
{\left[[\eta], j_{2} l_{2 n-5}\right] } & = \pm j_{2} P_{6} p_{6} i_{1} s_{6} \eta \\
& = \pm j_{2} P_{5} s_{5} \eta \\
& =0 \bmod 0
\end{aligned}
$$

If $n \equiv 2 \bmod 4$ the group $\pi_{2 n}\left(V_{2 n, 6}\right)=Z_{4}+Z_{2}+Z_{2}$ is generated by $i_{2}[v], i_{1}[\eta] \eta$ and $[\eta]$, so we may set

$$
s_{*}[\eta] \equiv[\eta] \bmod i_{5} v^{2}
$$

Because of $i_{1}[\eta]=H_{6} P_{1} l_{2 n+1}$ in $\pi_{2 n}\left(V_{2 n+1,6}\right)$, the relations $P_{5}\left[\eta_{2 n-1}\right]=P_{6} i_{1}[\eta]=0$ hold. Therefore

$$
\left[[\eta], j_{2} l_{2 n-5}\right]= \pm j_{2} P_{5}[\eta]=0
$$


We know that the group $\pi_{2 n+2}\left(W_{n, 3}\right)=Z_{4\left(b_{n}, 2\right)}+Z_{16}$, where the first component is generated by $\left[\left(2 /\left(b_{n}, 2\right)\right) v\right]$.

Theorem 8. We have $\left[\left[\left(2 /\left(b_{n}, 2\right)\right) v\right], j_{2} l_{2 n-5}\right]=0$, where the indeterminacy is $j_{2}\left[\sigma, l_{2 n-5}\right]$ when $n \neq 2 \bmod 8$.

Proof. Let $k=3, l=1$ and $r=2 n+1$ in (1.1). In the case $n \equiv 2 \bmod 4$, the group $\pi_{2 n+2}\left(V_{2 n, 6}\right)=Z_{4}+Z_{4}+Z_{4}$ is generated by $i_{4} s_{2} \sigma, i_{3}\left[v^{2}\right]$ and $[2 l] v$, so we may set

$$
s_{*}[2 v] \equiv[2 \imath] v \bmod i_{4} s_{2} \sigma, 2 i_{3}\left[v^{2}\right]
$$

by $(1.2)_{4}$. Since $E P_{2}\left[v_{2 n-4}^{2}\right]=\left[v^{2}, l_{2 n-4}\right]=E^{3} P_{4}\left[v^{2}\right]$ and $H_{1} P_{4}\left[v^{2}\right]=v^{3}$ hold, $P_{2}\left[v^{2}\right]$ is the unstable element $P_{5}[2 i] v$ of the group $\pi_{4 n-4}^{2 n-5}$. By

$$
\begin{aligned}
\pm b_{n} v_{2 n-5^{*}} \pi_{4 n-4}^{2 n-2} & \supset \pm b_{n} v_{2 n-5^{*}} E^{3} \pi_{4 n-7}^{2 n-5} \\
& = \pm b_{n} v_{4 n-7}^{*} \pi_{4 n-7}^{2 n-5} \\
& \ni v_{4 n-7}^{*} P_{5}[2 l] \\
& =P_{5}[2 l] v
\end{aligned}
$$

we have $P_{2}\left[v_{2 n-4}^{2}\right]=P_{5}[2 l] v \in$ Image $\Delta_{2}$. Thus

$$
\begin{aligned}
{\left[[2 v], j_{2} l_{2 n-5}\right] } & \equiv \pm j_{2} P_{6} p_{6} i_{1}[2 l] v \bmod j_{2} P_{6} p_{6} i_{5} s_{2} \sigma \\
& = \pm j_{2} P_{6} p_{6} i_{4}\left[v^{2}\right] \\
& = \pm j_{2} P_{2}\left[v^{2}\right] \\
& \equiv 0 \bmod j_{2}\left[\sigma, l_{2 n-5}\right] .
\end{aligned}
$$

Especially in the case $n \equiv 2 \bmod 8$, we have $\left[\sigma, l_{2 n-5}\right]=0$.

Let $n \equiv 0 \bmod 4$. Since the 2 components of the group $\pi_{2 n+2}\left(V_{2 n, 6}\right)$ is $Z_{4}+Z_{4}+Z_{8}$ generated by $i_{4} s_{2} \sigma, i_{3}\left[v^{2}\right]$ and $s_{6} v$, we have

$$
s_{*}[v] \equiv s_{6} v \bmod i_{4} s_{2} \sigma, 2 i_{3}\left[v^{2}\right]
$$

If $n \equiv 4 \bmod 8$ we have

$$
\begin{aligned}
{\left[[v], j_{2} l_{2 n-5}\right] } & = \pm j_{2} P_{5} s_{5} v_{2 n-1} \\
& \equiv 0 \bmod j_{2}\left[\sigma, l_{2 n-5}\right],
\end{aligned}
$$


since $H_{8} P_{1} l_{2 n+3}=i_{3} s_{5} v$ implies $P_{5} s_{5} v=P_{8} i_{3} s_{5} v=0$. If $n \equiv 0 \bmod 8, n \geqq 24$, the relations $i_{8} \sigma=i_{4} s_{5} v$ and $\left[\sigma, i_{2 n-5}\right] \neq 0$ (cf. [3]) imply

$$
0 \neq P_{5} s_{5} v_{2 n-1}=\left[\sigma, l_{2 n-5}\right] \notin b_{n} v_{2 n-5} * \pi_{4 n-4}^{2 n-2} \text {. }
$$

Hence

$$
\begin{aligned}
{\left[[v], j_{2} l_{2 n-5}\right] } & = \pm j_{2} P_{5} s_{5} v_{2 n-1} \\
& \equiv 0 \bmod j_{2}\left[\sigma, l_{2 n-5}\right] .
\end{aligned}
$$

Let $(l: m)=l / l, m)$, where $(l, m)$ is the greatest common measure of the integers $l$ and $m$. We have $\pi_{2 n+6}\left(W_{n, 3}\right)=Z_{16\left(4: b_{n}\right)}+Z_{2\left(4: b_{n}\right)}+Z_{2}$, where $Z_{16\left(4: b_{n}\right)}$ is generated by $[\sigma]$.

Theorem 9. We have $\left[[\sigma], j_{2} l_{2 n-5}\right]=0$ for $n \cong 0,6 \bmod 8, \quad\left[[\sigma], j_{2} l_{2 n-5}\right]=$ $\pm j_{2} P_{5} s_{5} \sigma_{2 n-1} \neq 0$ for $n \equiv 4 \bmod 8$ (the indeterminacy is $j_{2}\left[\zeta, l_{2 n-5}\right]$ when $n \equiv 60 \bmod 64$ ), $\left[[\sigma], j_{2} l_{2 n-5}\right]= \pm j_{2} P_{5}\left[\sigma_{2 n-1}\right] \neq 0$ for $n \equiv 2 \bmod 8$.

Proof. Let $k=3, l=3$ and $r=2 n+5$ in (1.1). For the case $n \equiv 0 \bmod 4$, the group $\pi_{2 n+6}\left(V_{2 n, 6}\right)=Z_{4}+Z_{8}+Z_{16}$ is generated by $i_{1}[\bar{v}], i_{1}[\eta] \sigma$ and $s_{6} \sigma$, so we may set $s_{*}[\sigma]=s_{6} \sigma$.

If $n \equiv 0 \bmod 8$ we have

$$
\begin{aligned}
{\left[[\sigma], j_{2} l_{2 n-5}\right] } & = \pm j_{2} P_{5} s_{5} \sigma_{2 n-1} \\
& \equiv 0 \bmod j_{2}\left[\zeta, l_{2 n-5}\right]=0,
\end{aligned}
$$

since $H_{12} P_{1} l_{2 n+7}=i_{7} s_{5} \sigma$ implies $P_{5} s_{5} \sigma_{2 n-1}=0$ and $\left[\zeta, l_{2 n-5}\right]=8 P_{5} s_{5} \sigma=0$.

If $n \equiv 4 \bmod 8$, we have

$$
\left[[\sigma], j_{2} l_{2 n-5}\right]= \pm j_{2} P_{5} s_{5} \sigma_{2 n-1} \bmod j_{2}\left[\zeta, l_{2 n-5}\right]=0
$$

by $\left[\zeta, i_{2 n-5}\right]=8 P_{5} s_{5} \sigma=0$ (except $\left[\zeta, l_{2 n-5}\right] \neq 0$ when $n \equiv 60 \bmod 64$ ). Since $E^{4} P_{5} s_{5} \sigma_{2 n-1}$ $=\left[\sigma, l_{2 n-1}\right]=E^{7} P_{8} s_{8} \sigma$ and $H_{1} P_{8} s_{8} \sigma=\sigma^{2}$ hold, $P_{5} s_{5} \sigma$ is the unstable element.

Assume that $P_{5} s_{5} \sigma_{2 n-1} \in \pm b_{n} v_{2 n-5} \pi_{4 n}^{2 n-2}$, then we have

$$
E P_{5} s_{5} \sigma_{2 n-1} \in \pm b_{n} v_{2 n-4} E \pi_{4 n}^{2 n-2}= \pm b_{n} v_{2 n-4} * \pi_{4 n+1}^{2 n-1}
$$

by the isomorphism $\pi_{4 n}^{2 n-2} \stackrel{E}{\rightarrow} \pi_{4 n+1}^{2 n-1}$. By the map $E^{3}: \pi_{4 n+1}^{2 n-4} \rightarrow \pi_{4 n+4}^{2 n-1}$, we have

$$
\begin{aligned}
0 \neq\left[\sigma, l_{2 n-1}\right] & =E^{4} P_{5} s_{5} \sigma_{2 n-1} \\
& \in \pm E^{3}\left(b_{n} v_{2 n-4} * \pi_{4 n+1}^{2 n-1}\right) \\
& = \pm b_{n} v_{4 n+1}^{*} \pi_{4 n+1}^{2 n-1}
\end{aligned}
$$


Here the unstable part $Z_{8}$ of the group $\pi_{4 n+1}^{2 n-1}$ generated by $P_{4}[\eta]$ (cf. [10]) vanishes by $b_{n} v_{4 n+1}^{*}$, since $\pm b_{n} v_{4 n+1}^{*} P_{4}[\eta]= \pm P_{4}\left(b_{n}[\eta] v\right)$ and $[\eta] v \in \pi_{2 n+6}\left(V_{2 n+3,4}\right)=Z_{2}+Z_{2}$. This is a contradiction.

Similarly assume $P_{5} s_{5} \sigma_{2 n-1} \in \eta_{2 n-5} \pi_{4 n}^{2 n-4}$. This leads to a contradiction as follows;

$$
0 \neq E^{7} P_{8}[\sigma] \in E^{4}\left(\eta_{2 n-5} \pi_{4 n}^{2 n-4}\right)=\eta_{4 n+3}^{*} E^{3} \pi_{4 n}^{2 n-4},
$$

where the group $\pi_{4 n+3}^{2 n-1}$ is stable. These show $P_{5} s_{5} \sigma_{2 n-1} \notin \operatorname{Image} \Delta_{2}$, therefore $j_{2} P_{5} s_{5} \sigma_{2 n-1} \neq 0$.

In the case $n \equiv 2 \bmod 4$, the group $\pi_{2 n+6}\left(V_{2 n, 6}\right)=Z_{4}+Z_{4}+Z_{32}$ is generated by $i_{1}[\bar{v}], i_{1}[\eta] \sigma-4[\sigma]$ and $[\sigma]$, so we have

$$
s_{*}[\sigma] \equiv[\sigma] \bmod i_{4} s_{2} \zeta .
$$

If $n \equiv 6 \bmod 8$, we have

$$
\left[[\sigma], j_{2} l_{2 n-5}\right]= \pm j_{2} P_{5}\left[\sigma_{2 n-1}\right]=0
$$

because the relation $H_{12} P_{1} l_{2 n+7}=i_{7}[\sigma]+i_{3} s_{9} v$ implies

$$
\begin{aligned}
P_{5}[\sigma] & =-P_{9} s_{9} v_{2 n+3} \\
\in & \pm b_{n} v_{4 n-3}^{*} \pi_{4 n-3}^{2 n-5} \\
& \subset \pm b_{n} v_{2 n-5^{*}} \pi_{4 n}^{2 n-2}
\end{aligned}
$$

If $n \equiv 2 \bmod 8$, we have

$$
\left[[\sigma], j_{2} l_{2 n-5}\right]= \pm j_{2} P_{5}\left[\sigma_{2 n-1}\right] .
$$

Assume $P_{5}\left[\sigma_{2 n-1}\right] \in \pm b_{n} v_{2 n-5} \pi_{4 n}^{2 n-2}$, then we have

$$
\begin{aligned}
E P_{5}\left[\sigma_{2 n-1}\right] & \in \pm b_{n} v_{2 n-4^{*}} E \pi_{4 n}^{2 n-2} \\
& = \pm b_{n} v_{2 n-4^{*}} \pi_{4 n+1}^{2 n-1}
\end{aligned}
$$

by the isomorphism $E: \pi_{4 n}^{2 n-2} \rightarrow \pi_{4 n+1}^{2 n-1}$. Here the unstable part $Z_{16}$ of the group $\pi_{4 n+1}^{2 n-1}$ vanishes by $v_{4 n+1}^{*}$, since

$$
\begin{aligned}
\pm b_{n} E^{3} v_{2 n-4^{*}} P_{5} s_{5} l_{2 n+3} & = \pm b_{n} v_{4 n+1}^{*} P_{5} s_{5} l_{2 n+3} \\
& = \pm b_{n} P_{5} s_{5} v \\
& = \pm b_{n} P_{8} i_{3} s_{5} v \\
& = \pm b_{n} P_{8} H_{8} P_{1} l_{2 n+7} \\
& =0 .
\end{aligned}
$$

This contradicts $E^{4} P_{5}\left[\sigma_{2 n-1}\right]=\left[\sigma, l_{2 n-1}\right] \neq 0$. 
Assume that $P_{5}\left[\sigma_{2 n-1}\right] \in \eta_{2 n-5^{*}} \pi_{4 n}^{2 n-4}$. Since the relations

$$
E^{4} \eta_{2 n-5^{*}} \pi_{4 n}^{2 n-4}=\eta_{4 n+3}^{*} E^{3} \pi_{4 n}^{2 n-4} \subset \eta_{4 n+3}^{*} \pi_{4 n+3}^{2 n-1}
$$

hold and the unstable part $Z_{2}$ of the group $\pi_{4 n+3}^{2 n-1}$ vanishes by $\eta_{4 n+3}^{*}$, we have a contradiction to $E^{7} P_{8}[\sigma]=\left[\sigma, l_{2 n-1}\right] \neq 0$. Therefore $P_{5}\left[\sigma_{2 n-1}\right] \notin$ Image $\Delta_{2}$. Hence, $j_{2} P_{5}\left[\sigma_{2 n-1}\right] \neq 0$.

\section{Whitehead products in $\pi_{r}\left(W_{n, 3}\right)$ for $n$ odd}

Let $n$ be odd. The group $\pi_{2 n-1}\left(W_{n, 3}\right)=Z$ is generated by $\left[\left(24 / a_{n}\right) l\right]$.

Theorem 10. $\left[\left[\left(24 / a_{n}\right) l\right] \cdot j_{2} l_{2 n-5}\right]=0$.

Proof. Let $l=1, k=3$ and $r=2 n-2$ in (1.1). Since $\pi_{2 n-1}\left(V_{2 n+1,7}\right)=0$ we obtain the result.

We know the 2 components of the group $\pi_{2 n+2}\left(W_{n, 3}\right)=Z_{4\left(a_{n}, 2\right)}+Z_{16}$, where the first component is generated by $\left[\left(2 /\left(a_{n}, 2\right)\right) v\right]$.

Theorem 11. We have $\left[\left[\left(2 /\left(a_{n}, 2\right)\right) v\right], j_{2} l_{2 n-5}\right]= \pm j_{2} P_{5}\left[v_{2 n-1}\right] \not \equiv 0 \bmod j_{2}\left[\sigma, l_{2 n-5}\right]$, $j_{2} P_{2}\left[v^{2}\right]$ if $n \equiv 3 \bmod 4, \equiv 0 \bmod j_{2}\left[\sigma, l_{2 n-5}\right], j_{2} P_{2}\left[v_{2 n-4}^{2}\right]$ if $n \equiv 1 \bmod 4$.

Proof. Let $l=1, k=3$ and $r=2 n+1$ in (1.1). In the case $n \equiv 1 \bmod 4$ the group $\pi_{2 n+2}\left(V_{2 n, 6}\right)=Z_{2}+Z_{2}+Z_{2}+Z_{2}+Z_{4}$ is generated by $i_{5} \bar{v}, i_{5} \varepsilon, i_{4} s_{2} \sigma, i_{3}\left[v^{2}\right]$ and $[2 l] v$, so we may set

$$
s_{*}[2 v] \equiv[2 l] v \bmod i_{5} \bar{v}, i_{5} \varepsilon, i_{4} s_{2} \sigma, i_{3}\left[v^{2}\right]
$$

in $(1.2)_{2}$. Thus

$$
\begin{aligned}
{\left[[2 v], j_{2} l_{2 n-5}\right] } & = \pm j_{2} P_{6} p_{6} i_{1}[2 l] v \\
& \equiv 0 \bmod j_{2}\left[\sigma, l_{2 n-5}\right], j_{2} P_{2}\left[v_{2 n-4}^{2}\right]
\end{aligned}
$$

because of $i_{1}[2 l] v \equiv 0 \bmod i_{6} \bar{v}, i_{6} \varepsilon, i_{5} s_{2} \sigma, i_{4}\left[v^{2}\right]$. Assume that $P_{2}\left[v_{2 n-4}^{2}\right] \in a_{n} v_{2 n-5^{*}} \pi_{4 n-4}^{2 n-2}$. Then we have the relations

$$
\eta_{4 n-4}^{*} P_{2}\left[v^{2}\right]=P_{2}\left[v^{2}\right] \eta=P_{6}[\eta] \eta^{2}=\left[\varepsilon, l_{2 n-5}\right] \neq 0
$$

This contradicts $\eta_{4 n-4}^{*} a_{n} v_{2 n-5} \pi_{4 n-4}^{2 n-2}=0$. Therefore $0 \neq P_{2}\left[v_{2 n-4}^{2}\right] \notin$ Image $\Delta_{2}$, so $j_{2} P_{2}\left[v^{2}\right] \neq 0$. A similar argument shows that $j_{2}\left[\sigma, l_{2 n-5}\right] \neq 0$ by $\eta_{4 n-4}^{*}\left[\sigma, l_{2 n-5}\right]$ $=\left[\eta \sigma, l_{2 n-5}\right] \neq 0$.

In the case $n \equiv 3 \bmod 4$, the group $\pi_{2 n+2}\left(V_{2 n, 6}\right)=Z_{2}+Z_{2}+Z_{2}+Z_{2}+Z_{8}$ is generated by $i_{5} \bar{v}, i_{5} \varepsilon, i_{4} s_{2} \sigma, i_{3}\left[v^{2}\right]$ and $[v]$, so we may set

$$
s_{*}[v] \equiv[v] \bmod i_{5} \bar{v}, i_{5} \varepsilon, i_{4} s_{2} \sigma, i_{3}\left[v^{2}\right]
$$


in (1.2) 2 . Since the unstable parts $Z_{2}+Z_{2}$ of the group $\pi_{4 n-4}^{2 n-2}$ generated by $P_{2} s_{2} l_{2 n-1}$ and $\left[\eta, l_{2 n-2}\right]$ (cf. [10]) vanish by the map $a_{n} v_{2 n-5^{*}}$, so the unstable elements $P_{5}\left[v_{2 n-1}\right]$, $P_{1} \sigma_{2 n-5}$ and $P_{2}\left[v_{2 n-4}^{2}\right]$ do not lie in Image $\Delta_{2}$. Thus

$$
\begin{aligned}
{\left[[v], j_{2} l_{2 n-5}\right] } & = \pm j_{2} P_{6} p_{6} i_{1}[v] \\
& = \pm j_{2} P_{5}\left[v_{2 n-1}\right] \\
& \neq 0 \bmod _{2}\left[\sigma, l_{2 n-5}\right], j_{2} P_{2}\left[v^{2}\right] .
\end{aligned}
$$

We know the 2 components of the group $\pi_{2 n+6}\left(W_{n, 3}\right)=Z_{16\left(4: a_{n}\right)}+Z_{2\left(a_{n}, 4\right)}$, where $Z_{16\left(4: a_{n}\right)}$ is generated by $[2 \sigma]$.

Theorem 12. $\left[[2 \sigma], j_{2} l_{2 n-5}\right]=0$.

Proof. Let $l=1, k=3$ and $r=2 n+5$ in (1.1). Since $\pi_{2 n+6}\left(V_{2 n+1,7}\right)=0$, the result follows.

\section{REFERENCES}

1. C. S. Hoo and M. E. Mahowald, Some homotopy groups of Stiefel manifolds, Bull. Amer. Math. Soc. 71 (1965), 661-667.

2. I. M. JAMEs, Note on Stiefel manifolds I, Bull. London Math. Soc. 2 (1970), 199-203.

3. L. Kristensen and I. Madsen, Note on Whitehead products in spheres, Math. Scand. 21 (1967), 301-314.

4. G. S. McCarTY, $\mathrm{J}_{\mathrm{R}}$, Products between homotopy groups and the $J$-morphism, Quart. $J$. Math. Oxford (2) 15 (1964), 362-370.

5. Y. Nomura, Some homotopy groups of real Stiefel manifolds in the metastable range I-V, Sci. Rep. Coll. Gen. Ed. Osaka Univ. 27 (1978), 1-31, 55-97; 28 (1979), 1-26, 35-60; 29 (1980), 159-183.

6. Y. Nomura and Y. Furukawa, Some homotopy groups of complex Stiefel manifolds, Sci. Rep. Coll. Gen. Ed. Osaka Univ. 25 (1976), 1-17.

7. Y. Nomura and Y. Furukawa, Whitehead products in $\pi_{r}\left(X_{n, 2}\right)$, to appear.

8. G. F. PAEChTER, The groups $\pi_{r}\left(V_{n, m}\right)$ I-V, Quart. J. Math. Oxford (2) 7 (1956), 249-268; 9 (1958), 8-27; 10 (1959), 17-37, 241-260; 11 (1960), 1-16.

9. F. Sigrist, Groupes d'homotopie des variétés de Stiefel complexes, Comment. Math. Helv. 43 (1968), 121-131.

10. S. Thомегеr, Einige Ergebnisse über Homotopiegruppen von Sphären, Math. Ann. 164 (1966), 225-250.

Department of Mathematics

AichI UNIVERSITY OF EDUCATION

KARIYA, JAPAN 448 